\title{
Symbolische Gewalt
}

\author{
Stephan Moebius • Angelika Wetterer
}

Die Frage nach den Mechanismen „Symbolischer Gewalt“ gehört zu den Schwerpunkten der soziologischen Analysen von Pierre Bourdieu. Der Erfolg dieses „Schlüsselkonzepts der bourdieuschen Soziologie" (Schultheis 2008) rührt insbesondere daher, dass es Bourdieu auf eindrucksvolle Weise gelang, die Kämpfe um symbolische Herrschaft und die Prozesse symbolischer Gewalt nicht nur theoretisch und empirisch, sondern auch für unterschiedliche soziale Felder analysiert zu haben, sei es im Kunstbetrieb (vgl. Bourdieu et al. 1981), im religiösen Feld (vgl. Bourdieu 2000), in der Justiz (vgl. Bourdieu 1986), im akademischen Feld (vgl. Bourdieu 1988a, 1998), im Geschlechterverhältnis (vgl. Bourdieu 2005a), in der Sprache (Bourdieu 2005b), in der Philosophie (vgl. Bourdieu 1988b), in der Politik (Bourdieu und Schwibs 1992, S. 174 ff.), in den Distinktionsbemühungen des Lebensstils (vgl. Bourdieu 1982) oder im literarischen Feld (vgl. Bourdieu 1999). Symbolische Gewalt ist ,allen gesellschaftlichen Beziehungen strukturell immanent" (Moebius und Peter 2009a, S. 28).

Bourdieu greift mit seinem Konzept der symbolischen Gewalt auf unterschiedliche soziologische Theoretiker wie Marx, Weber, Elias, Durkheim oder Mauss zurück, wobei die Besonderheit seines Konzepts im Vergleich zu den Klassikern darin liegt, dass er weit mehr als diese auch die körperliche und unbewusst-spontane Dimension von symbolischer Gewalt sowie ihren objektiven Charakter in den Blick nimmt (vgl. Schmidt 2009, S. 232). Kennzeichnend für die symbolische Gewalt ist, dass sie auf der symbolisch-sinnhaften Ebene des Selbstverständlichen und Alltäglichen operiert und zur Bejahung, Verinnerlichung und Verschleierung von gesellschaftlichen Herrschaftsverhältnissen führt (vgl. Moebius 2011). Die symbolische Gewalt - ein Ausdruck, den Bourdieu synonym zu symbolischer Macht oder symbolischer Herrschaft gebraucht (zur Problematisierung dieser unscharfen Verwendung der Termini siehe die Beiträge von Lothar Peter und Beate Krais in diesem Heft) - ist vor allem über Kultur, das heißt über die symbolischen Dimensionen des sozialen Lebens, die Sinnbezüge, die Weltan-

(C) VS Verlag für Sozialwissenschaften 2011

Univ.-Prof. Dr. S. Moebius $(\bowtie) \cdot$ Univ.-Prof. Dr. A. Wetterer Institut für Soziologie, Karl-Franzens-Universität Graz, Universitätsstraße 15, $8010 \mathrm{Graz}$, Österreich

E-Mail: stephan.moebius@uni-graz.at

Univ.-Prof. Dr. A. Wetterer

E-Mail: angelika.wetterer@uni-graz.at 
sichten und selbstverständlichen Denkweisen vermittelt. „Beide Seiten, die Machthaber und die Machtunterworfenen, müssen über dasselbe Deutungs- und Bewertungssystem verfügen, damit symbolische Gewalt wirksam werden kann“. (Peter 2004, S. 49) Nur so kann die symbolische Gewalt eine unanzweifelbare Geltung in der Wahrnehmung der Menschen erlangen: „Von symbolischer Herrschaft oder Gewalt sprechen heißt davon, dass der Beherrschte, von einem subversiven Aufruhr abgesehen, der zur Umkehrung der Wahrnehmungs- und Bewertungskategorien führt, dazu tendiert, sich selbst gegenüber den herrschenden Standpunkt einzunehmen." (Bourdieu 2005a, S. 202) Das heißt, die Dominierten tragen paradoxerweise zu ihrer eigenen Unterwerfung bei. Die symbolische Gewalt hat deshalb eine gleichsam ,magische“ Kraft (Bourdieu 2001, S. 216 f.); sie ist das Ergebnis einer ,magischen“ Verwandlung von objektiver gesellschaftlicher Macht in symbolische Macht, die Herrschaftsbestrebungen und Machtpositionen als ihr vermeintliches Gegenteil erscheinen lässt, sei es als selbstlose Liebe (Kirchen und Wohlfahrtsverbände), als natürliche Gegebenheit (wie im Falle der ,,männlichen Herrschaft“) oder als „durch Zufall“" erlangte Errungenschaften. ${ }^{1}$

$\mathrm{Zu}$ den Grundeigenschaften symbolischer Gewalt gehört, dass ihr Repressionsgehalt weder unmittelbar bewusst wird noch offen zutage tritt. Sie ist eine ,sanfte Gewalt", eine Herrschaft über die „Köpfe und Herzen“ (Krais 2004, S. 186), die ihren Herrschaft stabilisierenden Effekt in erster Linie dadurch erlangt, dass sie qua Sozialisation - in den Worten Foucaults (1978) - das „Körperinnere durchzieht“. Aktualisiert und verbreitet wird die symbolische Gewalt insbesondere in Kultur produzierenden Institutionen wie im öffentlichen Bildungswesen, in Kirchen, Parlamenten und Medien, in Literatur- und Kunstbetrieben sowie in den Wissenschaften, in Institutionen also, die für gewöhnlich den Ruf genießen, zweck- oder besonders herrschaftsfreie Gebiete zu sein. Dabei ist die Sprache - auch im physischen Sinne als „Technik des Körpers“ (Marcel Mauss) verstanden (Bourdieu und Wacquant 1996, S. 184) - ein zentrales Medium symbolischer Macht und Gewalt (vgl. Bourdieu und Wacquant 1996, S. 175 ff., 2005b, c), da durch sie die Klassifikationen, Bedeutungssetzungen und Sinngebungen performativ erzeugt und die Machtverhältnisse reproduziert werden (vgl. Bourdieu und Schwibs 1992, S. 153). Besonders anschaulich zeigt Bourdieu die symbolische Macht der Sprache in seiner soziologischen Studie zur Heidegger'schen Philosophie (Bourdieu 1988b; Bourdieu und Wacquant 1996, S. 185 ff.), aber auch in der Analyse eines Interviews mit dem damaligen Präsidenten der Deutschen Bundesbank, Hans Tietmeyer, das die Tageszeitung Le Monde im Oktober 1996 veröffentlichte. ${ }^{2}$ Bourdieu zitiert und kommentiert dieses Interview folgendermaßen:

,Deshalb müssen die öffentlichen Haushalte unter Kontrolle gehalten werden und das Steuer- und Abgabenniveau auf ein langfristig erträgliches Niveau gesenkt, das soziale Sicherungssystem reformiert und die Starrheiten des Arbeitsmarkts abge-

1 Vgl. etwa die Studie von Monique de Saint Martin (2003) über den Adel, der seine gesellschaftliche Machtposition sich selbst damit erklärt, dass er sie entweder aus eigener Leistung oder durch Zufall erworben habe. Die Prominenz und medialen Selbstperformances von Freiherr zu Guttenberg würden ein exzellentes Beispiel einer weiteren Analyse in dieser Richtung abgeben. Siehe dazu auch jüngst den instruktiven Aufsatz von Soeffner (2011).

2 Hier wird auf Moebius (2011, S. 63 ff.) zurückgegriffen. Siehe auch Peter (2004, S. 68 ff.). 
baut werden, denn wir werden nur dann wieder eine neue Wachstumsphase erleben, wenn wir' - dieses ,wir' ist herrlich [eine Anmerkung Bourdieus] - ,wenn wir auf dem Arbeitsmarkt eine Flexibilisierungsanstrengung vollbringen'. Das wär's. [...] Eine vorzügliche rhetorische Leistung, die folgendermaßen übersetzt werden kann: Nur Mut, liebe Arbeiter! Vollbringen wir diese gemeinsame Flexibilisierungsanstrengung, die von euch gefordert wird! (Bourdieu 2003, S. 185 f.)

Die neoliberale Anforderung, auf den „Arbeitsmärkten Flexibilisierungsanstrengungen zu vollbringen“ übersetzt Bourdieu: „Gebt heute eure sozialen Errungenschaften auf, um das Vertrauen der Investoren nicht zu gefährden, und dies zugunsten eines Wachstums, welches uns morgen zugute kommt“ (Bourdieu 2003, S. 186). Die symbolische Macht neoliberaler Rhetorik ist nach Bourdieu bereits so sehr im Alltag verbreitet und verinnerlicht, dass sie ,perfekt in den ,Erwartungshorizont" der meisten Tageszeitungsleser passt“ (Bourdieu 2003, S. 187). Man liest Bourdieu zufolge über die zitierten Zeilen und die von Tietmeyer intendierten Ziele so leicht hinweg, weil sie mittlerweile zur Struktur unserer inkorporierten Denk- und Beurteilungsschemata geworden sind. Begriffe wie „Flexibilität“, „dauerhaftes Wachstum“, „Vertrauen der Investoren“ oder „Globalisierung“ gelten inzwischen als allgemeine Basistugenden bzw. Verhaltensanforderungen, die sich nicht nur auf den Finanzmarkt beschränken. Sie sind zu kollektiven und kontextübergreifenden Werten geworden:

Dieser sich ökonomisch gebende Diskurs kann sich nur dann über die engeren Kreise seiner Urheber hinaus verbreiten, wenn er auf die Mitarbeit einer großen Anhängerschaft aus Politikern, Journalisten und einfachen Bürgern zählen kann, die über einen ausreichend ökonomischen Anstrich verfügen, um sich an der allgemeinen Verbreitung ungenügend definierter Begriffe aus einer ökonomischen Vulgata beteiligen zu können. (Bourdieu 2003, S. 188)

So liegt die symbolische Gewalt des neoliberalen Diskurses darin, dass die hinter Begriffen wie „Flexibilität“ oder „dauerhaftes Wachstum“ liegenden Machtverhältnisse unklar werden und die Beherrschten sie zunehmend mit positiven Erwartungen besetzen. Es findet eine Verklärung von Machtbeziehungen zu Sinnbeziehungen statt (vgl. Peter 2004).

In einem Interview mit Effi Böhlke spezifiziert Bourdieu sein Konzept folgendermaßen:

Um das Problem der symbolischen Macht und ihrer spezifischen Wirksamkeit adäquat zu stellen, muss man m. E. die traditionelle Frage nach den Beziehungen zwischen Ökonomie und den anderen ,Instanzen' konsequent fallenlassen. Die symbolische Macht ist eine Macht, die jedesmal ausgeübt wird, wenn eine Macht (oder ein Kapital) ökonomischer oder auch physischer (die Kraft als Zwangsinstrument), kultureller oder sozialer Art in die Hände von Agenten gelangt, deren Wahrnehmungs- und Bewertungskategorien den Strukturen dieser Macht, oder genauer gesagt, ihrer Verteilung angepasst sind und die daher dazu neigen, sie als natürlich, als selbstverständlich wahrzunehmen und die ihr zugrundeliegende willkürliche Gewalt zu verkennen, sie also als legitim anzuerkennen. (Bourdieu 2007, S. 265) 
„Verkennen“ meint nach Bourdieu:

[...] den Tatbestand, eine Gewalt anzuerkennen, die genau in dem Maße ausgeübt wird, wie man sie als Gewalt verkennt; den Tatbestand also, jenes Ensemble der grundlegenden, vor-reflexiven Voraussetzungen $\mathrm{zu}$ akzeptieren, die die sozialen Akteure schon dadurch mitmachen, daß sie die Welt als etwas Selbstverständliches nehmen, das heißt so, wie es ist, und sie natürlich finden, weil sie kognitive Strukturen auf sie anwenden, die aus eben diesen Strukturen der Welt hervorgegangen sind. (Bourdieu und Waquant 1996, S. 205)

Wirkt die symbolische Gewalt durch (unbewusste) Ver-undAnerkennung der Beherrschten, durch eine Art „Beziehung hingenommener Komplizenschaft, die bewirkt, daß bestimmte Aspekte dieser Welt stets jenseits oder diesseits kritischer Infragestellung stehe" (Bourdieu 2005c, S. 82), so werden aber auch die Herrschenden von der symbolischen Gewalt beherrscht. So schreibt Bourdieu (2004, S. 16) in seiner bildungssoziologischen Studie über den Staatsadel:

Wenn es richtig ist, an den Eigenbeitrag der Beherrschten zur Herrschaft zu erinnern, so ist sogleich daran zu erinnern, daß die Dispositionen, die sie zu dieser Mitwirkung verleiten, gleichfalls ein - inkorporierter - Herrschaftseffekt sind, genauso wie, nebenbei bemerkt, die Dispositionen, die dafür verantwortlich sind, daß, nach einem Wort von Marx, ,die Herrschenden von ihrer Herrschaft beherrscht‘ werden. (Siehe dazu auch Beate Krais in diesem Heft.)

Fasst man die wesentlichen Punkte symbolischer Gewalt zusammen (vgl. Mauger 2005, S. 218 ff.), dann wirkt sie erstens vornehmlich durch Sprache, Kommunikationsbeziehungen sowie durch Denk- und Wahrnehmungsschemata. Ausgeübt wird sie zweitens durch Gesten, Rituale, Verhaltensweisen und Dinge. Hierbei geht es in einer Art „Amnesie der Entstehungsgeschichte der symbolischen Gewalt" vor allem um die Verschleierung, Kaschierung und Naturalisierung der Machtverhältnisse (z. B. die Naturalisierung der männlichen Herrschaft), woraufhin die Macht legitimiert wird. Drittens setzt symbolische Gewalt voraus, dass die Machtverhältnisse, auf denen die Gewalt beruht, verkannt und zugleich „die Prinzipien, in deren Namen sie ausgeübt wird, anerkannt werden“ (Mauger 2005, S. 218).

Um die Mechanismen symbolischer Gewalt adäquat aus den Alltagsvorstellungen und -praktiken herauszudestillieren, sie analysieren und interpretieren sowie kritisch hinterfragen zu können, bedarf es eines durch die soziologische Analyse herbeigeführten Bruchs mit dem Alltagsverständnis (der doxa), wie Bourdieu et al. (1991) in ihrem wissenschaftstheoretischen Buch Soziologie als Beruf ausführlich darlegen. Wie bereits der Wissenschaftshistoriker Gaston Bachelard hervorgehoben hat, auf den Bourdieus Überlegungen zum Bruch mit der „Spontansoziologie“ beruhen (vgl. Moebius und Peter 2009b), lassen sich erst durch diesen Bruch neue wissenschaftliche Erkenntnisse zu Tage fördern und gesellschaftliche Herrschaftsverhältnisse offen legen (vgl. auch Bourdieu und Wacquant 1996, S. 205). Deshalb beanstandet Bourdieu an der gegenwärtigen Soziologie nicht nur eine dem Alltagsverständnis verwandte Sichtweise der sozialen Welt, sondern kritisiert auch jene „Spielarten“ der Soziologie, die nur deshalb auf den ersten Blick eine besondere Evidenz erlangen, weil sie ,ihre wissenschaftliche Fragestellung 
um Termini aus dem vertrauten Wortschatz der Alltagssprache aufbauen“ (Bourdieu et al. 1991, S. 26). In der Soziologie seien Mischtermini oder Mischschemata besonders beliebt, die ihre „pseudo-explikative Leistung“ genau dieser doppelten Zugehörigkeit zu den ,naiven wie wissenschaftlichen Äußerungen“ verdanken und ,ihre Herkunft aus der Umgangssprache unter dem Zierrat des wissenschaftlichen Jargons" kaschieren (Bourdieu et al. 1991, S. 27) - man denke etwa an Begriffe wie „Massen-“, „Informations-““ oder „Kommunikationsgesellschaft“.

Die Bourdieu'schen Analysen der Prozesse symbolischer Gewalt zeigen eindrucksvoll die Notwendigkeit eines solchen epistemologischen Bruchs mit dem Alltagsverständnis und führen damit die Notwendigkeit und Aktualität kritischer Soziologie vor Augen (vgl. Peter 2006a) - einer kritischen Soziologie, die sich nicht nur auf eine Explikation der kritischen Haltungen der sozialen Akteure im Alltag, auf eine „Soziologie der Kritik“ (Boltanski und Thévenot 2007; vgl. auch Basaure 2008; Bogusz 2010, S. 127 ff.) also, beschränken mag, ${ }^{3}$ sondern tiefer schürft, in dem sie eine Kritik des Selbstverständlichen und (angeblich) natürlich Gegebenen vornimmt, die den Herrschaftscharakter gesellschaftlicher Prozesse und Strukturen erkennbar macht und jene Begriffe, Denk-, Wahrnehmungs- und Verhaltensschemata kritisch dekonstruiert, „die gesellschaftliche Herrschaft ausblenden, verharmlosen, verschleiern oder rechtfertigen. “(Peter 2006b)

Wirft man einen Blick auf die gegenwärtigen deutschsprachigen Rezeptionen des Konzepts symbolischer Gewalt, so lassen sich insbesondere folgende Rezeptionslinien ausmachen: Neben theoretischen Vertiefungen, Systematisierungen und Diskussionen des Konzepts (vgl. etwa Neckel 2002; Peter 2004; Mauger 2005; Rehbein 2006; Celikates 2009; Moebius 2011; Schmidt 2009) existieren erstaunlicherweise immer noch relativ wenige Forschungen und Studien, die das Konzept empirisch anwenden (vgl. Schmidt 2009, S. 234). Die derzeit vorliegenden Arbeiten kommen vornehmlich aus der Frauenund Geschlechterforschung (zuerst: Krais 1993; vgl. des Weiteren Dölling und Krais 1997; Bock et al. 2007, S. 63-140) sowie aus einer geschlechtersoziologisch orientierten Bildungs- und Wissenschaftssoziologie (vgl. etwa Engler 1993; Engler 2001; Beaufays 2003). Einen entscheidenden Schritt in Richtung einer gesellschaftsanalytischen Anwendung stellt in jüngerer Zeit der Sammelband Symbolische Gewalt. Herrschaftsanalyse nach Pierre Bourdieu, herausgegeben von Schmidt und Woltersdorff (2008) dar. Neben werkgeschichtlichen und theoretischen Aufsätzen zur Genese des bourdieuschen Schlüsselkonzepts (Schultheis 2008) oder zur Herrschaft sichernden Funktionsweise symbolischer Gewalt (Krais 2008) finden sich dort auch Beiträge, die das Konzept hinsichtlich bildungs-, wissenschafts-, medien-, politik- sowie arbeitssoziologischer Perspektiven diskutieren und zur Anwendung bringen. Mit der Produktivität des Bourdieu'schen Denkens für die Kulturwissenschaften befassen sich schließlich die Beiträge in dem jüngst erschienenen, breit angelegten Sammelband Bourdieu und die Kulturwissenschaften Prinz/Schäfer/Šuber), der in Teil III (S. 157-201) Untersuchungen und Analysen vorstellt, die sich mit der symbolischen Gewalt in medialen Repräsentationen befassen: Repräsentationen von Klasse und Geschlecht im Hollywoodfilm (Prinz und Clauss 2011), Konstruktio-

3 Zur Diskussion um unterschiedliche, aktuelle soziologische Modelle der Kritik (Bourdieu, Boltanski, kritische Theorie) siehe das instruktive Buch von Celikates (2009) sowie den Beitrag von Voswinkel \& Wagner in diesem Heft. Zur Soziologie als Gesellschaftskritik siehe auch Moebius und Schäfer (2006) und Peter (2006a, b). 
nen des Fremden in Banlieu-Berichterstattung und popkulturellen Darstellungen (Keller 2011), symbolische Gewalt aber auch in aktuellen Diskursen um Neo- und Antifeminismus (Dölling 2011).

Bourdieus Konzept der symbolischen Gewalt weist große Ähnlichkeiten mit anderen derzeit in der Soziologie und den Kulturwissenschaften verstärkt debattierten Konzeptionen gesellschaftlicher Macht- und Herrschaftsverhältnisse auf; Konzeptionen, wie sie etwa in Anschluss an Michel Foucaults Begriff der produktiven Macht in den Gouvernementalitätsforschungen (governmentality studies) und in poststrukturalistischen Sozialwissenschaften (vgl. Moebius 2008) sowie in den Diskussionen um einen „Neuen Geist des Kapitalismus“ (Boltanski und Chiapello 2003) ausgearbeitet wurden. Wie das Konzept der symbolischen Gewalt thematisieren auch diese Machtkonzeptionen Formen von Macht und Gewalt, die nicht in erster Linie über (physischen) Zwang ausgeübt werden, sondern über diskursive und nicht-diskursive Verfahren der Ausrichtung der Körper, der identifikatorischen Bindung an bestimmte, von der symbolischen Ordnung vorgegebene Identitäten, der Produktion bestimmter Subjektpositionen und kognitiver Ordnungsschemata (Episteme, Wissen), die alternative Deutungs- und Wissensmuster als nicht denkbar, sichtbar und sagbar erscheinen lassen, sowie über die Modellierung der Zeit-Raum-Vorstellungen, der Praktiken der Selbstbildung und der Wahrnehmungs- und Verhaltensschemata.

Inwiefern ist das Bourdieu'sche Konzept der symbolischen Gewalt mit diesen neueren Konzeptionen zu vereinbaren? Sind es ähnliche Konzepte, die allenfalls unter einem anderen Namen diskutiert werden? Oder kann man die unterschiedlichen Machtkonzeptionen als komplementär betrachten? Welche Potenziale für die soziologische Forschung hält Bourdieus Konzept noch bereit? Wo sind etwaige Kritiken an diesem Konzept vorzubringen? In welchem Verhältnis steht Bourdieus herrschaftskritische Soziologie zu den gegenwärtigen Diskussionen um eine „Soziologie der Kritik“?

Ausgehend von diesen Fragen will das vorliegende Themenschwerpunktheft der ÖZS Bourdieus Konzeption der symbolischen Gewalt näher beleuchten. Besonders ertragreich für ein solches Vorhaben erschien es uns, die Produktivität dieser Konzeption zum einen im Theorievergleich herauszuarbeiten und sie zum anderen anhand der Untersuchung ausgewählter sozialer Bereiche auszuloten, in denen die symbolische Gewalt ihre „magische“ Kraft entfaltet. Nach einem einführenden Beitrag von Lothar Peter, der Bourdieus Überlegungen im Rahmen theorie- und begriffsgeschichtlicher Entwicklungen vorstellt und zu systematisieren und weiterzuentwickeln sucht, wendet sich Beate Krais den Geschlechterverhältnissen und der männlichen Herrschaft zu. Ihr Beitrag zeichnet unter Rekurs auf Bourdieus Habitus-Konzept nach, worauf die verdeckte Gewaltförmigkeit inkorporierter Denk-, Wahrnehmungs- und Handlungsschemata beruht und weshalb die männliche Herrschaft mit Bourdieu als paradigmatische Form symbolischer Gewalt zu begreifen ist, der die Beherrschten ebenso unterliegen wie die Herrschenden. Gleichsam kontrapunktisch fragt Paula-Irene Villa im nächsten Schritt nicht nach der ,magischen“ Kraft, sondern nach den Möglichkeiten der Kritik und des Scheiterns symbolischer Gewalt. Villa vergleicht Bourdieus Konzept symbolischer Gewalt mit Judith Butlers Überlegungen zu Sprache, Gewalt und Subjektkonstitution und findet erst bei Butler systematische Ansatzpunkte dafür, die magischen Verblendungen konterkarieren zu können, die diskursiv verfassten Vergesellschaftungs- und Anerkennungsverhältnissen 
inhärent sind. In anderer Weise um die Frage nach den Möglichkeiten der Kritik geht es in dem Beitrag von Gabriele Wagner und Günter Voswinkel; für sie stellt sich diese Frage in einem gesellschaftstheoretischen und gegenwartsdiagnostischen Rahmen. Wagner \& Voswinkel konstatieren für die Gegenwart eine allgemeine Sprachlosigkeit der Kritik am Kapitalismus, die sie in Anlehnung an die Überlegungen zum „Neuen Geist des Kapitalismus“"von Boltanski \& Chiapello auf die symbolische Macht gegenwärtiger Individualisierungssemantiken und Rechtfertigungsordnungen zurückführen, angesichts derer eine Erneuerung der Kritik überfällig sei, wie sie allererst eine reflexive Verbindung von kritischer Soziologie und Soziologie der Kritik leisten könn(t)e. Auch im letzten Beitrag des Heftes werden Bourdieus Überlegungen zur symbolischen Gewalt im Theorievergleich erörtert. Anne Waldschmidt fragt danach, wie sich die soziale Konstruktion von (Nicht-)Behinderung soziologisch denken lässt und zeichnet nach, welche je verschiedenen Facetten dieses Konstruktionsprozesses in den Blick kommen, wenn man ihn im Anschluss an Goffmans Stigmatheorie, im Rahmen von Foucaults Diskurs- und Machttheorie oder mit Bourdieu als Effekt symbolischer Gewalt untersucht. Ihr Fazit, mit Bourdieu ließe sich Behinderung als ,eine in ihrem Herrschaftscharakter gründlich verkannte, weil legitimierte Devianz“ (S. 12) denken, weist am Schluss dieses Heftes auf die herrschaftskritischen Potenziale hin, die Bourdieus Denken - allen diesbezüglichen Zweifeln zum Trotz - bis heute inhärent sind.

Das vorliegende Themenheft zur „Symbolischen Gewalt" könnte deshalb, wie wir hoffen, vor allem für die zukünftigen Theoriedebatten gewinnbringend sein, die verstärkt nach einem soziologisch produktiven, die unausgesprochenen, impliziten und oft unerkannten gesellschaftlichen Herrschaftsverhältnisse aufdeckenden Analysekonzept von Macht suchen und Soziologie nicht nur als l'art pour l'art betreiben, sondern sie als kritische, auf der Ebene wissenschaftlicher Standards operierende Reflexion und Problematisierung gesellschaftlicher Herrschaftsverhältnisse verstehen, die immer auch abzielt auf eine Offenlegung der sozialen, ökonomischen, geschlechtlichen und ethnischen Ungleichheiten.

\section{Literatur}

Basaure, Mauro. 2008. Die pragmatistische Soziologie der Kritik heute. Mauro Basaure im Gespräch mit Luc Boltanski. Berliner Journal für Soziologie 18 (4): 526-549.

Beaufays, Sandra. 2003. Wie werden Wissenschaftler gemacht? Beobachtungen zur wechselseitigen Konstitution von Geschlecht und Wissenschaft. Bielefeld: transcript.

Bock, Ulla, Irene Dölling, und Beate Krais, Hrsg. 2007. Prekäre Transformationen. Pierre Bourdieus Soziologie der Praxis und ihre Herausforderung für die Frauen- und Geschlechterforschung. Göttingen: Wallstein.

Bogusz, Tanja. 2010. Zur Aktualität von Luc Boltanski. Einleitung in sein Werk, Reihe: Aktuelle und klassische Sozial- und KulturwissenschaftlerInnen. Wiesbaden: VS Verlag für Sozialwissenschaften.

Boltanski, Luc, und Eve Chiapello. 2003. Der neue Geist des Kapitalismus. Konstanz: UVK.

Boltanski, Luc, und Laurent Thévenot. 2007. Über die Rechtfertigung. Eine Soziologie der kritischen Urteilskraft. Hamburg: Hamburger Edition.

Bourdieu, Pierre. 1982. Die feinen Unterschiede. Kritik der gesellschaftlichen Urteilskraft. Frankfurt a. M.: Suhrkamp. 
Bourdieu, Pierre. 1986. La force du droit. Actes de la recherche en sciences sociales 64:5-19.

Bourdieu, Pierre. 1988a. Homo academicus. Frankfurt a. M.: Suhrkamp.

Bourdieu, Pierre. 1988b. Die politische Ontologie Martin Heideggers. Frankfurt a. M.: Suhrkamp.

Bourdieu, Pierre. 1998. Vom Gebrauch der Wissenschaft. Für eine klinische Soziologie des wissenschaftlichen Feldes. Konstanz: UVK.

Bourdieu, Pierre. 1999. Die Regeln der Kunst. Genese und Struktur des literarischen Feldes. Frankfurt a. M.: Suhrkamp.

Bourdieu, Pierre. 2000. Genese und Struktur des religiösen Feldes. In Das religiöse Feld. Texte zur Ökonomie des Heilsgeschehens, Hrsg. Ders., 39-110. Konstanz: UVK.

Bourdieu, Pierre. 2001. Meditationen. Zur Kritik der scholastischen Vernunft. Frankfurt a. M.: Suhrkamp.

Bourdieu, Pierre. 2003. Das Modell Tietmeyer. In absolute Pierre Bourdieu, Hrsg. Joseph Jurt, 184-189. Freiburg i. Br.: orange press.

Bourdieu, Pierre. 2004. Der Staatsadel. Konstanz: UVK.

Bourdieu, Pierre. 2005a. Die männliche Herrschaft. Frankfurt a. M.: Suhrkamp.

Bourdieu, Pierre. 2005b. Was heißt sprechen? Zur Ökonomie des sprachlichen Tausches. 2. Aufl. Wien: Braumüller.

Bourdieu, Pierre. 2005c. Die verborgenen Mechanismen der Macht enthüllen. In Die verborgenen Mechanismen der Macht. Schriften zu Politik \& Kultur 1, Hrsg. Ders., 81-86. Hamburg: VS Verlag für Sozialwissenschaften.

Bourdieu, Pierre. 2007. Politisches Feld und symbolische Macht. Gespräch mit Effi Böhlke. In Bourdieu und die Linke. Politik - Ökonomie - Kultur, Hrsg. Effi Böhlke und Rainer Rilling, 263-270. Berlin: Dietz.

Bourdieu, Pierre, und Bernhard Schwibs. 1992. Rede und Antwort. Frankfurt a. M.: Suhrkamp.

Bourdieu, Pierre, und Loïc Wacquant. 1996. Reflexive Anthropologie. Frankfurt a. M.: Suhrkamp.

Bourdieu, Pierre, et al. 1981. Eine illegitime Kunst. Die sozialen Gebrauchsweisen der Photographie. Frankfurt a. M.: EVA.

Bourdieu, Pierre, et al. 1991. Soziologie als Beruf. Wissenschaftstheoretische Voraussetzungen soziologischer Erkenntnis. Berlin: de Gruyter.

Celikates, Robin. 2009. Kritik als soziale Praxis. Gesellschaftliche Selbstverständigung und kritische Theorie. Frankfurt a. M.: Campus.

De Saint Martin, Monique. 2003. Der Adel. Soziologie eines Standes. Konstanz: UVK.

Dölling, Irene. 2011. Symbolische Gewalt in aktuellen Diskursen zum Anti- bzw. Neo-Feminismus. In Bourdieu und die Kulturwissenschaften. Zur Aktualität eines undisziplinierten Denkens, Hrsg. Sophie Prinz et al., 179-197. Konstanz: UVK.

Dölling, Irene, und Beate Krais, Hrsg. 1997. Ein alltägliches Spiel. Geschlechterkonstruktionen in der sozialen Praxis. Frankfurt a. M.: Suhrkamp.

Engler, Steffani. 1993. Fachkultur, Geschlecht und soziale Reproduktion. Eine Untersuchung über Studentinnen und Studenten der Erziehungswissenschaft, Rechtswissenschaft, Elektrotechnik und des Maschinenbaus. Weinheim: Deutscher Studienverlag.

Engler, Steffani. 2001. ,In Einsamkeit und Freiheit'? Zur Konstruktion der wissenschaftlichen Persönlichkeit auf dem Weg zur Professur. Konstanz: UVK.

Foucault, Michel. 1978. Die Machtverhältnisse durchziehen das Körperinnere. In Dispositive der Macht. Über Sexualität, Wissen und Wahrheit, Hrsg. Ders., 104-117. Berlin: Merve.

Keller, Carsten. 2011. Mediale Konstruktion des Fremden: Die französische Banlieu im Spiegel von Berichterstattungen und popkulturellen Darstellungen. In Bourdieu und die Kulturwissenschaften. Zur Aktualität eines undisziplinierten Denkens, Hrsg. Sophie Prinz et al., 199-215. Konstanz: UVK.

Krais, Beate. 1993. Geschlechterverhältnis und symbolische Gewalt. In Praxis und Ästhetik. Neue Perspektiven im Denken Pierre Bourdieus, Hrsg. Gunter Gebauer und Christoph Wulf, 208-250. Frankfurt a. M.: Suhrkamp. 
Krais, Beate. 2004. Soziologie als teilnehmende Objektivierung der sozialen Welt: Pierre Bourdieu. In Französische Soziologie der Gegenwart, Hrsg. Stephan Moebius und Peter Lothar, 171-210. Konstanz: UVK.

Krais, Beate. 2008. Zur Funktionsweise von Herrschaft in der Moderne: Soziale Ordnungen, symbolische Gewalt, gesellschaftliche Kontrolle. In Symbolische Gewalt. Herrschaftsanalyse nach Pierre Bourdieu, Hrsg. Robert Schmidt und Volker Woltersdorff, 45-58. Konstanz: UVK.

Mauger, Gérard. 2005. Über symbolische Gewalt. In Bourdieu, Pierre: Deutsch-französische Perspektiven, Hrsg. Cathérine Colliot-Thélène et al., 208-230. Frankfurt a. M.: Suhrkamp.

Moebius, Stephan. 2008. Macht und Hegemonie: Grundrisse einer poststrukturalistischen Analytik von Macht. In Poststrukturalistische Sozialwissenschaften, Hrsg. Stephan Moebius und Andreas Reckwitz, 158-174. Frankfurt a. M.: Suhrkamp.

Moebius, Stephan. 2011. Pierre Bourdieu: Zur Kultursoziologie und Kritik der symbolischen Gewalt. In Kultur. Theorien der Gegenwart. 2. erweiterte, aktualisierte und überarbeitete Auflage, Hrsg. Stephan Moebius und Dirk Quadflieg, 55-69. Wiesbaden: VS Verlag für Sozialwissenschaften.

Moebius, Stephan, und Gerhard Schäfer, Hrsg. 2006. Soziologie als Gesellschaftskritik. Wider den Verlust einer aktuellen Tradition. Hamburg: VS Verlag für Sozialwissenschaften.

Moebius, Stephan, und Lothar Peter, Hrsg. 2009a. Pierre Bourdieu und der Strukturalismus. In Bourdieu-Handbuch, Hrsg. Gerhard Fröhlich und Boike Rehbein, 20-28. Stuttgart: J. B. Metzler.

Moebius, Stephan, und Lothar Peter, Hrsg. 2009b. Pierre Bourdieu und die französische Epistemologie. In Bourdieu-Handbuch, Hrsg. Gerhard Fröhlich und Boike Rehbein, 10-15. Stuttgart: J. B. Metzler.

Neckel, Sieghard. 2002. Die Mechanismen symbolischer Macht. Kabylen und Kapitalismus: Einführendes zur Soziologie Pierre Bourdieus. In Theorie als Kampf? Zur politischen Soziologie Pierre Bourdieus, Hrsg. Uwe Bittlingsmayer et al., 29-34. Opladen: Leske \& Budrich.

Peter, Lothar. 2004. Pierre Bourdieus Theorie der symbolischen Gewalt. In Pierre Bourdieu. Politisches Forschen, Denken und Eingreifen, Hrsg. Margareta Steinrücke, 48-73. Hamburg: VS Verlag für Sozialwissenschaften.

Peter, Lothar. 2006a. Soziologie als Herrschaftskritik - Wissenschaft und Politik bei Pierre Bourdieu. In Willkürliche Grenzen. Das Werk Pierre Bourdieus in interdisziplinärer Perspektive, Hrsg. Mark Hillebrand et al., 21-32. Bielefeld: transcript.

Peter, Lothar. 2006b. Wozu noch Gesellschaftskritik? Blätter für deutsche und internationale Politik 5:587-599.

Prinz, Sophie, Hilmar Schäfer, und Daniel Suber, Hrsg. 2011. Bourdieu und die Kulturwissenschaften. Zur Aktualität eines undisziplinierten Denkens. Konstanz: UVK.

Prinz, Sophia, und Mareike Clauss. 2011. ,A head for business and a bod' for sin. ' Klasse und Geschlecht im Hollywood-Frauenfilm. In Bourdieu und die Kulturwissenschaften. Zur Aktualität eines undisziplinierten Denkens, Hrsg. Sophie Prinz et al., 157-178. Konstanz: UVK.

Rehbein, Boike. 2006. Die Soziologie Pierre Bourdieus. Konstanz: UVK.

Schmidt, Robert. 2009. Symbolische Gewalt. In Bourdieu-Handbuch, Hrsg. Gerhard Fröhlich und Boike Rehbein, 231-235. Stuttgart: J. B. Metzler.

Schmidt, Robert, und Volker Woltersdorff, Hrsg. 2008. Symbolische Gewalt. Herrschaftsanalyse nach Pierre Bourdieu. Konstanz: UVK.

Schultheis, Franz. 2008. Symbolische Gewalt: Zur Genese eines Schlüsselkonzepts der bourdieuschen Soziologie. In Symbolische Gewalt. Herrschaftsanalyse nach Pierre Bourdieu, Hrsg. Robert Schmidt und Volker Woltersdorff, 25-44. Konstanz: UVK.

Soeffner, Hans-Georg. 2011. Fassadenpolitik. Ein Baron spielt den Bürger. Soziologie. Forum der Deutschen Gesellschaft für Soziologie 40 (3): 267-275. 
Stephan Moebius, geb. 1973, ist Universitätsprofessor für Soziologische Theorie und Ideengeschichte am Institut für Soziologie der Karl-Franzens-Universität Graz. Er ist Sprecher der Sektion Kultursoziologie der Deutschen Gesellschaft für Soziologie und Sprecher der Sektion Soziologische Theorie der Österreichischen Gesellschaft für Soziologie. Forschungsschwerpunkte: Soziologiegeschichte, Kultursoziologie, Soziologische Theorie, Religionssoziologie. Publikationen (Auswahl): Kultur. Theorien der Gegenwart (2. Auflage, hrsg. mit D. Quadflieg), Wiesbaden: VS 2011; Kultur, Bielefeld: transcript 2010 (2. Aufl.); Soziologische Kontroversen. Beiträge zu einer anderen Geschichte der Wissenschaft vom Sozialen (hrsg. mit G. Kneer), Frankfurt/M.: Suhrkamp 2010 .

Angelika Wetterer, promovierte Germanistin und habilitierte Soziologin, ist Professorin für Soziologie der Geschlechterverhältnisse und Leiterin des Bereichs Geschlechtersoziologie \& Gender Studies am Institut für Soziologie der Karl-Franzens-Universität Graz. Forschungsschwerpunkte: Professionalisierung, Arbeitsteilung \& Geschlechterkonstruktion; feministische Wissenschafts- und Gesellschaftstheorie; Modernisierungsprozesse im Geschlechterverhältnis; Frauen- \& Gleichstellungspolitik in praktischer und theoretischer Perspektive. 